\title{
Uso de marcadores genotoxicológicos para la evaluación de agricultores expuestos a plaguicidas organofosforados
} Use of genotoxic markers for the evaluation of farmers exposed to organophosphorous

\author{
Jaime Rosales ${ }^{1, a, b}$ \\ Centro Nacional de Salud Ocupacional y Protección del Ambiente para la Salud. Instituto Nacional de Salud. Lima, Perú. \\ ${ }^{\mathrm{a}}$ Tecnólogo Médico, ${ }^{\mathrm{b}}$ Magíster en Salud Ocupacional.
}

\begin{abstract}
Resumen
Introducción. Los plaguicidas organofosforado son compuestos utilizados para el control de plagas en actividades agrícolas; genera exposición ocupacional asociada a posibles intoxicaciones agudas y crónicas que se manifiestan por alteraciones a nivel bioquímico, molecular y genético, los cuales pueden ser evaluados mediante el uso de marcadores genotoxicológicos. Objetivo. Determinar el uso de marcadores genotoxicológicos para la evaluación de agricultores expuestos a plaguicidas organofosforados. Diseño. Observacional, analítico, transversal con grupo control. Lugar. Empresa de agroexportación de espárragos en el Distrito de Virú, La Libertad. Intervenciones. Se determinó la actividad enzimática de la colinesterasa sérica (BChE) y eritrocitaria (AChE), y se hizo ensayos de genotoxicidad (aberraciones cromosómicas, ensayo cometa y micronúcleos) en 59 trabajadores expuestos a plaguicidas (grupo expuesto) y 50 personas sin exposición a plaguicidas (grupo control). Principales medidas de resultados. Actividad enzimática de la colinesterasa sérica (BChE) y eritrocitaria (AChE), y ensayos de genotoxicidad (aberraciones cromosómicas, ensayo cometa y micronúcleos). Resultados. Las edades promedio del grupo expuesto y control fueron de 39,6 $\pm 10,8$ y $34,0 \pm 11,5$ años, respectivamente. En referencia a la actividad BChE, se encontró diferencia significativa $(p<0,001)$ entre el grupo expuesto $(4733.0 \pm 1350.1 \mathrm{U} / \mathrm{L})$ y control (7 075.0 $\pm 1674.0 \mathrm{U} / \mathrm{L})$. Caso contrario se presentó en la actividad AChE entre el grupo expuesto $(4867,0 \pm 632,2 \mathrm{U} / \mathrm{L})$ y control (5 051,0 $\pm 505,5 \mathrm{U} / \mathrm{L}$ ). Además, se evidenció la inhibición de ambas enzimas en 9 trabajadores expuestos por debajo del valor de referencia. No se evidenció diferencias significativas en los marcadores genotoxicológicos, a excepción del recuento de micronúcleos puentes nucleoplásmicos, gemaciones, binucleaciones celulares y cariorexis de células de epitelio bucal; y en la evaluación del daño al ADN mediante el ensayo cometa $(p<0,05)$ se obtuvo promedios de intensidad de cola de $8,28 \pm 1,85$ (Rango: 6,06-15,75) y $7,30 \pm 1,25$ (Rango. 4,04-9,41) para el grupo expuesto y control, respectivamente. Conclusiones. El uso de marcadores genotoxicológicos aportó información relevante como herramienta que permite predecir el riesgo asociado a cáncer, considerando que el evento inicial es el daño al ácido desoxiribonucléico (ADN) en cualquier etapa del ciclo celular; además, existió asociación significativa entre el efecto genotóxico y la exposición a plaguicidas organofosforados evidenciada por la inhibición de la BChE.
\end{abstract}

Palabras clave. Insecticidas organofosforados; Colinesterasa sérica; Colinesterasa eritrocitaria; Ensayo cometa; Micronúcleos; Aberraciones cromosómicas.

\begin{abstract}
Introduction: Organophosphorous pesticides are compounds used to control pests in agricultural activities. They generate potential occupational exposure associated with acute and chronic poisoning manifested by biochemical, molecular and genetic alterations that may be assessed using genotoxic markers. Objective: To evaluate genotoxicologic markers use for assessing farmers exposed to organophosphate pesticides. Design: Observational, analytical, cross-sectional study with control group. Institution: Asparagus Agro Exportation Company in District of Virú, La Libertad. Interventions: Enzyme activity of serum (BChE) and erythrocyte cholinesterase (AChE), and genotoxicity assays (chromosome aberrations, comet assay and micronucleus) were assessed in 59 workers exposed to pesticides (exposed group) and 50 individuals not exposed to pesticides (control group). Main outcome measures: Enzyme activity of serum (BChE) and erythrocyte cholinesterase (AChE), and genotoxicity assays (chromosome aberrations, comet assay and micronucleus). Results: Average age of the exposed and control groups were respectively $39.6 \pm 10.8$ and $34.0 \pm 11.5$ years. Referring to BChE activity, significant difference $(p<0.001)$ was found between the exposed group $(4733.0 \pm 1350.1 \mathrm{U} / \mathrm{L})$ and control group (7075.0 $\pm 1674.0 \mathrm{U} / \mathrm{L})$. The contrary occurred in the AChE activity between the exposed (4867.0 $\pm 632.2 \mathrm{U} / \mathrm{L})$ and control group $(5051.0 \pm 505.5 \mathrm{U} / \mathrm{L})$. Furthermore, there was evident inhibition of both enzymes below the reference value in 9 workers. No significant difference was observed in genotoxic markers, except for buccal epithelium cells micronuclei count, nucleoplasmic bridges, buddings, binucleation and karyorrhexis. In comet assay DNA damage evaluation $(p<0.05)$ mean queue intensity was $8.28 \pm 1.85$ (range: 6.06 15.75) and $7.30 \pm 1.25$ (range: 4.04-9.41) for the exposed and control groups, respectively. Conclusions: Use of genotoxicologic markers provided relevant information to predict cnacer-associated risk considering the initial event is damage to deoxyribonucleic acid (DNA) at any stage of the cell cycle. Significant association between genotoxic effects and exposure to organophosphorous pesticides was demonstrated by BChE inhibition.
\end{abstract}

Keywords: Insecticides, Organophosphate; Serum cholinesterase; Erythrocyte cholinesterase; Comet assay; Micronuclei; Chromosomal aberrations.

An Fac med. 2015;76(3):247-52 / http://dx.doi.org/10.15381/anales.v76i3.11233 


\section{INTRODUCCIÓN}

En el Perú, el auge de la exportación agrícola y el uso deficiente de medidas de protección en trabajadores agrícolas han incrementado los casos de intoxicación a plaguicidas. La Dirección General de Epidemiología del Ministerio de Salud (DGE-MINSA) registró 48730 casos de intoxicación aguda por plaguicidas en el periodo $2000-2013^{(1)}$.

La exposición laboral a plaguicidas puede generar intoxicaciones agudas con manifestaciones neurológicas tales como el síndrome clásico, intermedio y de neurotoxicidad retardada; e intoxicaciones crónicas con alteraciones neuropsicológicas.

Los agricultores se exponen a plaguicidas, sobre todo de la clase organofosforados, durante su preparación, aplicación, almacenaje o trabajo en el campo. Cuando ingresa el plaguicida organofosforado, este inhibe la actividad enzimática de la colinesterasa, alterando la función del sistema nervioso periférico y autónomo y en consecuencia, a los órganos que dependen de ellos, afectando finalmente al sistema nervioso central. La mayoría de plaguicidas son absorbidos por vía respiratoria, digestiva, dérmica y ocular lo cual determina su alta capacidad de ingreso al organismo, produciendo diferentes alteraciones fisiológicas con distinto grado de toxicidad según el tipo de plaguicida ${ }^{(2)}$.

La evaluación precoz de la toxicidad asociada a la exposición por plaguicidas es una actividad que comprende la implementación de un programa de vigilancia sanitaria específica, siendo importantes las acciones de monitoreo biológico por el laboratorio (mediante el uso de marcadores de exposición, efecto y susceptibilidad en el trabajador expuesto) ${ }^{(3)}$. Precisamente, una característica de la epidemiología molecular constituye el uso de marcadores biológicos que permitan medir la exposición a diversos agentes de riesgo, así como aquellos que protegen o favorecen el desarrollo de la enfermedad en estadios subclínicos ${ }^{(4)}$. Incluso en algunos plaguicidas que son clasificados como car- cinógenos según la IARC (International Agency for Research on Cancer) ${ }^{(5)}$ es relevante caracterizar el riesgo por la exposición a estos agentes, utilizando métodos que evalúen el efecto genotóxico producido. La vigilancia epidemiológica es una actividad fundamental en el control de riesgos asociados a la exposición ocupacional a plaguicidas en el sector agrícola; y en Perú, únicamente ha estado basada en la evaluación clínica orientada al hallazgo de síntomas y signos típicos de intoxicación aguda. Sin embargo, existe un grupo mayoritario de trabajadores que presentan exposición crónica a plaguicidas y que no son identificados debido a que las manifestaciones clínicas suelen presentarse a largo plazo, afectando la capacidad cognitiva, incluso asociándose a enfermedades neurodegenerativas y psiquiátricas, así como procesos de mutagénesis, carcinogénesis y teratogénesis ${ }^{(10)}$.

En nuestro país, la determinación de la actividad colinesterasa sérica representa el único marcador de efecto de uso conocido e incluso limitado ante la exposición a plaguicidas; por ende el objetivo del presente trabajo fue utilizar marcadores genotoxicológicos que estén orientados a evaluar el efecto temprano y tardío producido por la exposición a plaguicidas, mediante el ensayo cometa y el estudio de micronúcleos y aberraciones cromosómicas, respectivamente, además de los marcadores clásicos como las enzimas colinesterasas.

\section{MÉTODOS}

Se diseñó un estudio observacional, analítico de corte transversal con grupo control.

La población de estudio estuvo constituida por agricultores expuestos a plaguicidas organofosforados de una empresa de agroexportación de espárragos del Distrito de Virú, Departamento La Libertad; y personas sin exposición a plaguicidas del Hospital Provincial de Virú. Se estimó el tamaño de la muestra comparando proporciones de prevalencia en ambos grupos de estudio, basado en una proporción esperada en el grupo de expuestos del 30\%, con un nivel de confianza de $95 \%$ y poder de prueba de $80 \%$. La muestra obtenida fue de 59 y 50 personas para el grupo de expuestos y no expuestos, respectivamente. Se consideró un tiempo de exposición mínima de 2 años para enrolar participantes al grupo de expuestos y además fueron divididos según actividades de fumigación (fumigadores y no fumigadores). Los criterios de exclusión del estudio fueron personas con enfermedades degenerativas, hepáticas, anemia, cáncer, o que hubieran estado expuestas a rayos X durante el último mes. Estos datos fueron obtenidos mediante la aplicación de una ficha epidemiológica y evaluación médica.

Se colectaron muestras de sangre por punción venosa (4 $\mathrm{mL}$ en tubo de extracción al vacío sin anticoagulante, para la obtención de suero) para el análisis de la actividad de butirilcolinesterasa (BChE), acetilcolinesterasa (AChE) y los ensayos de genotoxicidad ( $4 \mathrm{~mL}$ en tubo de extracción al vacío con heparina sódica). También se obtuvieron células epiteliales por hisopado bucal, las cuales fueron usadas para el ensayo de micronúcleos. Para el caso de los fumigadores, estos fueron evaluados 24 horas después de haber culminado su actividad.

La actividad enzimática de la BChE fue medida usando un kit comercial (Wiener Lab) y las lecturas fueron realizadas en un autoanalizador bioquímico CM250-WienerLab con un método cinético enzimático. La actividad enzimática de la AChE fue medida según lo descrito por Ellman y col ${ }^{(6)}$. Se utilizó un espectrofotómetro Thermo Scientific modelo Genesys 300 para las lecturas de absorbancia a una longitud de onda de $405 \mathrm{~nm}$.

Se realizó el cultivo de linfocitos para la obtención de células en metafase. Se añadió $500 \mathrm{uL}$ de sangre heparinizada a 5 mL de RPMI 1640 tratado con 200 $\mathrm{uL}$ de fitohemaglutinina y enriquecido con $20 \%$ de suero bovino fetal y glutamina. Los tubos fueron homogenizados 
y puestos a incubación a $37^{\circ}$ a $5 \% \mathrm{CO}_{2}$ por 48 horas; 90 minutos antes de la cosecha, se añadió Colcemid 0,2 ug/mL. La cosecha, fijación, tinción y lectura se procedieron de acuerdo a lo indicado por la IAEA (International Atomic Energy Agency) (7).

Las células obtenidas por hisopado bucal fueron fijadas en Carnoy y sometidas a 3 lavados consecutivos, previa centrifugación a $1200 \mathrm{rpm}$ por $10 \mathrm{mi}$ nutos. Se realizó preparados en láminas portaobjetos limpios con alcohol absoluto y secados al aire. Las células fueron teñidas con Giemsa al 2\% diluido en buffer fosfato de Sorensen pH 7,2 (El buffer se preparó mezclando $49 \mathrm{~mL}$ de 0,06 M Na2HPO4 (8,52 g/L) con 51 $\mathrm{mL}$ de KH2PO4 0,06 M (8,16 g/L)). Se realizó un recuento de 2000 células epiteliales en un microscopio de luz visible a 100X. Se empleó los criterios para el reconocimiento de micronúcleos según lo descrito por Tolbert y col ${ }^{(8)}$.

Se usó el ensayo cometa para evaluar daño del ADN según lo descrito por Rojas y col ${ }^{(9)}$. Se prepararon láminas embebidas en $150 \mathrm{uL}$ de agarosa regular $0,5 \%$ y secadas a $60^{\circ} \mathrm{C}$ por 1 hora. Luego se mezcló 10 uL de sangre con 75 uL de agarosa bajo punto de fusión (LMPA) $0,5 \%$ a $37^{\circ} \mathrm{C}$ y se añadió a la lámina previamente preparada; se cubrió con una laminilla, para que se solidificara, entre $4-8^{\circ} \mathrm{C}$ por 5 minutos. Posteriormente, se retiró la laminilla cuidadosamente y se añadió $75 \mathrm{uL}$ de LMPA 0,5\% para cubrirse y solidificarse nuevamente a $4^{\circ} \mathrm{C}$ por 5 minutos. Una vez que la lámina estuvo preparada, fue sumergida en un buffer de lisis, $\mathrm{pH}=10$ (40 mL stock de lisis (146,1 g NaCl 2,5 M, 37,2 g EDTA disódico $100 \mathrm{mM}$ y $1,2 \mathrm{~g}$ Tris Base $10 \mathrm{mM}$, csp $1 \mathrm{~L}$ de agua ultrapura), 4 mL de DMSO y 400 uL de tritón X-100), por 1 hora a $4^{\circ} \mathrm{C}$. Luego se ubicó las láminas en el tanque de ensayo cometa para realizar el desenrrollamiento del ADN en el buffer de electroforesis por 20 minutos. Dicho buffer se preparó con $30 \mathrm{~mL} \mathrm{NaOH} 10 \mathrm{~N}$ y 5 mL EDTA disódico 200 mM csp, 1 L de agua ultrapura y ajustado con gotas de $\mathrm{NaOH} 10 \mathrm{~N}$ a un $\mathrm{pH}=13,1$. Los parámetros de electroforesis fueron a $25 \mathrm{~V}$ y $300 \mathrm{mAmp}$ por 20 minutos. El tanque de ensayo cometa estuvo conectado a un chiller para asegurar la corrida a $4^{\circ} \mathrm{C}$. Terminada la electroforesis, las láminas fueron trasladadas a un buffer de neutralización (48,5 g Tris base $0.4 \mathrm{M}$ en $1 \mathrm{~L}$ de agua ultrapura, ajustado a un $\mathrm{pH}=7,5$ ) por dos veces a 5 minutos; y fueron deshidratadas en etanol absoluto por 5 minutos, retiradas y puestas en posición vertical hasta su completo secado. Finalmente, se realizó la tinción con $20 \mathrm{uL}$ de bromuro de etidio $20 \mathrm{~g} / \mathrm{L}$ diluido 1:10. La lectura se realizó en un microscopio de epifluorescencia marca Meiji. La medición del parámetro del ensayo cometa (intensidad de cola) fue obtenida con el software Comet Assay IV de Perceptive Instrument Ltda. sobre los nucleoides obtenidos de células mononucleares de sangre periférica.

El análisis estadístico se realizó usando el software IBM SPSS v 19,0 año 2013. Se calculó la frecuencia y medidas de tendencia central presentados en tablas de doble entrada, prueba de chi cuadrado, t-student y prueba no paramétrica U de Mann-Whitney, previo análisis de normalidad con la prueba de Shapiro-Wilk. Se consideró como diferencia significativa un valor de $\mathrm{p}<0,05$.

Los participantes del estudio fueron informados de los procedimientos a realizar, riesgos, beneficios y confidencialidad de los resultados. Los participantes otorgaron y firmaron el consentimiento voluntariamente.

\section{RESULTADOS}

Las características sociodemográficas más relevantes en los grupos de estudio se muestran en la tabla 1 , donde las personas que no fueron fumigadores se dedicaban a actividades de sembrío, cosecha y envasado de vegetales para su exportación. También se incluyó el tiempo laboral y la presencia de hábitos usuales, como el consumo de alcohol y cigarrillos.

La tabla 2 resume las características laborales del grupo expuesto a plaguicidas, donde se muestra que la mayoría de fumigadores realizaban su labor con distintos sistemas de fumigación y además no utilizaban los equipos de protección personal de modo integral.

La tabla 2 muestra la actividad colinesterasa sérica y eritrocitaria, hallándose diferencia significativa entre los grupos de estudio $(\mathrm{p}<0,001)$ para el primer caso, mas no para el segundo. Sin embargo, tomando como referencia los valores normales para ambos mar-

Tabla 1. Características sociodemográficas de los grupos de estudio.

\begin{tabular}{|c|c|c|c|c|}
\hline & \multicolumn{2}{|c|}{ Grupo expuesto ( $n=59$ ) } & \multicolumn{2}{|c|}{ Grupo control $(n=50)$} \\
\hline & $x \pm D E$ (Rango) & Frecuencia (\%) & $x \pm D E$ (Rango) & Frecuencia (\%) \\
\hline Edad (en años) & $39,6 \pm 10,8(20$ a 66$)$ & --- & $34,0 \pm 11,5$ (19 a 68) & --- \\
\hline Género (varón-mujer) & $40-19$ & $67,8-32,2$ & $17-33$ & $34-66$ \\
\hline Fumigador & --- & 40,7 & --- & --- \\
\hline Tiempo laboral (en años) & $10,0 \pm 7,2$ (3 а 38) & --- & --- & --- \\
\hline Consumo de cigarrillos & --- & 1,7 & --- & 10,0 \\
\hline Consumo de alcohol & --- & 45,8 & --- & 30,0 \\
\hline
\end{tabular}


Tabla 2. Aspectos laborales en el grupo expuesto*.

\begin{tabular}{ccc} 
& \multicolumn{3}{c}{ Frecuencia (\%) } \\
\cline { 2 - 3 } Uso mixto de sistemas de fumigación a & Sí & No \\
Uso completo de EPP b & 66,7 & 33,3 \\
Come, bebe o fuma durante la fumigación & 16,7 & 83,3 \\
Cambio de ropa de trabajo al finalizar la fumigación & 29,2 & 70,8 \\
Baño después de la fumigación & 95,8 & 4,2 \\
Antecedentes de intoxicación por plaguicidas diagnosticada por médico** & 95,8 & 4,2 \\
\hline
\end{tabular}

* Análisis estadístico aplicado sólo a fumigadores que constituyen parte del grupo expuesto.

${ }^{* \star}$ Análisis estadístico aplicado al total de individuos que constituyen el grupo expuesto, considerando un mínimo de 6 meses antes de ocurrido la intoxicación.

${ }^{a}$ Bomba de espalda manual, nebulizadora tirada por tractor, bomba de espalda a motor y bomba tirada por tractor o automotor a pistón.

${ }^{b}$ EPP: Equipos de protección personal - Uso de mandil y gorro impermeable descartable, lentes de seguridad, respirador con filtros para aerosoles, guantes y botas descartables.

Tabla 3. Actividad colinesterasa en los grupos de estudio de estudio.

\begin{tabular}{|c|c|c|c|c|}
\hline Enzima & $\begin{array}{c}\text { Grupo expuesto } \\
\mathrm{n}(\%)\end{array}$ & $\begin{array}{c}\text { Grupo control } \\
\mathrm{n}(\%)\end{array}$ & $X \pm D E$ & ${ }^{*} \mathrm{p}$ \\
\hline \multicolumn{5}{|l|}{${ }^{1} \mathrm{BChE}(\mathrm{U} / \mathrm{L})$} \\
\hline $\begin{array}{l}<3269 \mathrm{U} / \mathrm{L} \\
\geq 3269 \mathrm{U} / \mathrm{L}\end{array}$ & $\begin{array}{c}9(15,3) \\
50(84,7)\end{array}$ & $\begin{array}{c}0(0,0) \\
50(100,0)\end{array}$ & $4733,0 \pm 1350,1^{* *}$ & 0,000 \\
\hline \multicolumn{5}{|l|}{${ }^{2} \mathrm{AChE}(\mathrm{U} / \mathrm{L})$} \\
\hline $\begin{array}{l}<4395 \mathrm{U} / \mathrm{L} \\
\geq 4395 \mathrm{U} / \mathrm{L}\end{array}$ & $\begin{array}{c}(15,3) \\
50(84,7)\end{array}$ & $\begin{array}{c}1(2,0) \\
49(98,0)\end{array}$ & $4867,0 \pm 632,2^{* *}$ & 0,193 \\
\hline
\end{tabular}

BChE: Butiril colinesterasa o colinesterasa sérica; ${ }^{2} \mathrm{AChE}$ : Acetil colinesterasa o colinesterasa eritrocitária.

* Probabilidad obtenida de la prueba t-student.

** Promedio y desviación estándar obtenidos solo del grupo expuesto.

cadores, se observa que $15,3 \%$ de los evaluados en el grupo expuesto presentó valores por debajo de dicho rango.

El uso de los marcadores genotoxicológicos se muestra en la tabla 4 , y se aprecia diferencias significativas entre los grupos de estudio para el ensayo de aberraciones cromosómicas y micronúcleos, mas no para el ensayo cometa.

\section{DISCUSIÓN}

La colinesterasa sérica (BChE, E.C. 3.1.1.8) y eritrocitaria (AChE, E.C. 3.1.1.7) son un grupo de enzimas con elevada tasa de variabilidad interindividual debido a factores como el peso, talla, sexo, edad y además por los polimorfismos genéticos asociados ${ }^{(11)}$. La más significativa que la eritrocitaria, la cual presenta una elevada correlación con las manifestaciones clínicas presentadas en personas con exposición aguda, como es el caso de las intoxicaciones ${ }^{(15)}$. Cabe señalar que en el presente estudio no se incluyó a mujeres gestantes ni personas con trastornos hepáticos y anemia previo análisis de transaminasas y hemograma; para asegurar que los datos obtenidos no estén sesgados por variables fisiológicas que afectan la actividad colinesterasa sérica ${ }^{(16)}$.

Las actividades de monitoreo biológico deben contar con información de la actividad basal de colinesterasa a fin de determinar el porcentaje de inhibición enzimática, el cual no debe sobrepasar el $30 \%$, después de haber realizado actividades que se asocien a la exposición a plaguicidas organofosforados y carbamatos, después de un periodo mínimo de 30 días ${ }^{(15)}$.

Una de las medidas de control de riesgos por exposición a los plaguicidas es el uso adecuado de los equipos de protección personal además de las buenas prácticas durante la jornada laboral. Nuestro estudio refleja en líneas generales que el personal expuesto no cumplía ninguna de las dos medidas señaladas; siendo precisamente los principales factores de riesgo que determinan el estado de salud en personal expuesto a plaguicidas ${ }^{(17)}$. El efecto temprano producido por la exposición a plaguicidas es variada, sin embargo una de las moléculas diana que interaccionan con los plaguicidas y sus metabolitos es el ADN, el cual es susceptible de sufrir rupturas en hebra simple o doble, que se puede manifestar a largo plazo con la presencia de aberraciones cromosómicas.

Los hallazgos que asocian exposición a plaguicidas y el efecto genotóxico son variables; incluso algunos autores no encontraron asociación significativa entre el daño genético estudiado a través del ensayo cometa, aberraciones cromosómicas y recuento de micronúcleos en células de epitelio bucal y su relación con la exposición a plaguici- 
Tabla 4. Marcadores genotoxicológicos en los grupos de estudio.

\begin{tabular}{|c|c|c|c|c|c|}
\hline & \multicolumn{2}{|c|}{ Grupo expuesto $=59$} & \multicolumn{2}{|c|}{ Grupo control $=50$} & \multirow{2}{*}{$\mathrm{p}$} \\
\hline & $x \pm S D$ & Frecuencia (\%) & $x \pm S D$ & Frecuencia (\%) & \\
\hline \multicolumn{6}{|l|}{ Aberraciones cromosómicas } \\
\hline Gap cromatídico & --- & 2,3 & --- & 0,0 & 0,291 \\
\hline Ruptura cromatídica con fragmento acéntrico & --- & 1,7 & --- & 0,0 & 0,541 \\
\hline Intercambio tri-radial & --- & 1,7 & --- & 2,0 & 0,709 \\
\hline Intercambio cuatri-radial & --- & 1,7 & --- & 0,0 & 0,709 \\
\hline Ensayo de micronúcleos & & & --- & & \\
\hline Recuento de micronúcleos & $0,34 \pm 0,86$ & 25,4 & $0,04 \pm 0,20$ & 4,0 & $0,009^{*}$ \\
\hline Puentes nucleoplásmicos & $0,31 \pm 0,53$ & 27,1 & $0,08 \pm 0,27$ & 8,0 & $0,006^{*}$ \\
\hline Gemaciones & $0,90 \pm 1,09$ & 54,2 & $0,22 \pm 0,47$ & 20,0 & $0,031^{*}$ \\
\hline Binucleación celular & $1,80 \pm 1,78$ & 66,1 & $0,78 \pm 1,04$ & 46,0 & $0,047^{*}$ \\
\hline Cariorexis & $0,64 \pm 1,17$ & 32,2 & $0,00 \pm 0,00$ & 0,0 & $0,002^{*}$ \\
\hline \multicolumn{6}{|l|}{ Ensayo cometa } \\
\hline Intensidad de cola & $8,28 \pm 1,85$ & --- & $7,30 \pm 1,25$ & --- & $0,0116^{* *}$ \\
\hline
\end{tabular}

* Probabilidad obtenida de la prueba Chi-cuadrado. **Probabilidad obtenida de la prueba U de Mann Whitney.

das ${ }^{(18)}$. Otros evidenciaron que la reparación del ADN de personas expuestas a plaguicidas no genera mayor alteración en comparación a un grupo control ${ }^{(19)}$. El recuento de micronúcleos en células binucleadas tampoco ha evidenciado diferencias significativas en el mismo grupo de expuestos evaluados bajo diseño longitudinal ${ }^{(20)}$.

El daño al ADN es un evento que se ha reportado en muchos estudios de investigación, habiéndose encontrado asociación significativa entre la exposición a plaguicidas y el daño al ADN usando el ensayo cometa aplicado a linfocitos obtenidos de sangre total de grupo expuesto y control ${ }^{(21)}$. Otros estudios de corte longitudinal también hallaron daño genético y cromosómico en personas expuestas a plaguicidas usando el ensayo cometa y el índice de aberraciones cromosómicas, respectivamente; sin embargo es importante mencionar que el daño basal encontrado disminuyó considerablemente en la etapa final del estudio, de tal modo que se puede inferir que el daño al ADN es un evento que de acuerdo factores externos como el uso correcto de equipos de protección personal, y factores internos como la condición fisiológica de cada persona puede activar sistemas de reparación del ADN que permiten revertir el daño ${ }^{(22)}$. Nuestros hallazgos son similares a otras investigaciones ya publicadas, en el cual se encontró daño al ADN evidenciado como un incremento de la intensidad de cola en personas expuestas a plaguicidas con diferencia significativa al grupo control. Dicho daño genotóxico incluso se evidencia usando otros parámetros del ensayo cometa como la longitud de cola ${ }^{(23)}$.

Sin embargo, es importante definir los criterios de exclusión en diseños que impliquen el uso de métodos genotoxicológicos, ya que el daño al ADN y su expresión a nivel cromosómico puede ser de origen multifactorial, incluso debido a condiciones como la edad, género, dieta, exposición médica a rayos $\mathrm{X}$, entre otros ${ }^{(24)}$.

La mayoría de estudios que han evaluado el efecto genotóxico por exposición a plaguicidas encontraron aumentos muy pequeños respecto a los grupos controles, y además la ausencia de marcadores de exposición hacen que las asociaciones no sean consistentes ${ }^{(25)}$.

La manifestación del efecto genotóxico puede estar condicionada por factores intrínsecos como la variabi- lidad de algunas enzimas que participan en los procesos de detoxificación y metabolismo de algunos plaguicidas. Por ejemplo, está comprobado que los polimorfismos genéticos de las enzimas GSTM1, GSTT1, GSTP1, CYP2E1 y EPHX1 están asociados a un incremento en el daño citogenético manifestado como aberraciones cromosómicas, micronúcleos e intercambio de cromátides hermanas aumentada (26). En nuestro estudio, se evidencia una clara diferencia en el recuento de micronúcleos de células de epitelio bucal en los grupos de estudio; sobre todo en aquellos que se dedican a actividades de fumigación, como ya se ha reportado en diversos estudios realizados $(27,28)$.

En conclusión, el uso de marcadores genotoxicológicos aporta información relevante como herramienta que permite predecir el riesgo asociado a cáncer, considerando que el evento inicial es el daño al ADN en cualquier etapa del ciclo celular; además, existe asociación significativa entre el efecto genotóxico y la exposición a plaguicidas evidenciada por la inhibición de la enzima colinesterasa sérica.

Como aspecto a considerar a futuro, es necesario evidenciar y caracterizar 
el deterioro cognitivo asociado a la exposición crónica a plaguicidas organofosforados a bajas dosis, aspecto que se presenta de modo muy usual en los trabajadores agrícolas; por lo que es importante se puedan aplicar pruebas neuropsicológicas que permitan la evaluación de la función sensorial, motora y sobre todo cognitiva de la persona; ya que el uso de colinesterasas no necesariamente pudieran estar inhibidas y correlacionar con exposiciones crónicas ${ }^{(29)}$.

Una alternativa de investigación a posteriori es la implementación de nuevos marcadores que evalúen el efecto por exposición crónica a bajas concentraciones de plaguicidas y su asociación con las manifestaciones clínicas a nivel neuropsicológico ${ }^{(30)}$.

\section{REFERENCIAS BIBLIOGRAFICAS}

1. Nayhua L. Situación epidemiológica de las intoxicaciones agudas por plaguicidas en Perú, 2012. Bol. Epidemiol (Lima). 2013;22(15):274-80.

2. Soreq H, Zakut H. Human cholinesterases and anticholinesterases. San Diego: Academic Press; 1993;14:314. Doi: 10.1046/j.1471-4159.1994.t011-63010390.x.

3. Lockridge O. Structure of human serum cholinesterase. BioEssays: news and reviews in molecular, cellular and developmental biology. 1988;9(4):125-8. Doi: 10.1002/bies.950090406

4. Collins AR. Molecular epidemiology in cancer research. Molec Aspects Med. 1998;(19):359-432.

5. International Agency for Research on Cancer. Occupational exposure in insecticide application and some pesticides. IARC Monographs on the Evaluation of Carcinogenic Risk to Humans (IARC, Lyon). 1991;53:179-250.

6. Ellman GL, Courtney DK, Andreas V, Featherstone RM. A new and rapid colorimetric determination of acetylcholinesterase activity. Biochem Pharmacol. 1961;(7):88-95. doi:10.1016/0006-2952(61)90145-9.

7. International Atomic Energy Agency. cytogenetic dosimetry: applications in preparedness for and response to radiation emergencies. 2011:53-9,

8. Tolbert PE, Shy CM, Allen JW. Micronuclei and other nuclear anomalies in buccal smears: a reid test in snuff users. Am J Epidemiol. 1991;134(8):840-50.

9. Rojas E, López MC, Valverde M. Single cell gel electrophoresis assay: methodology and applica- tions. J Chromatography B. 1999;722:225-54. Doi: 10.1016/S0378-4347(98)00313-2.

10. Ntzani EE, Chondrogiorgi M, Ntritsos G, Evangelou $E$, Tzoulaki I. Literature review on epidemiological studies linking exposure to pesticides and health effects. EFSA supporting publication. 2013:EN497.

11. Brock A, Brock V. Factor affecting inter-individual variation in human plasma cholinesterase activity: Body weight, height, sex, genetic polymorphism and age. Arch Envirom Contam Toxicol. 1993;(24):93-9.

12. Maroto IM., Ramírez F, Zúñiga CM. Vega S. Variabilidad intraindividual de la actividad de las colinesterasas sanguíneas en una muestra humana costarricense. Rev Cost Cienc Méd. 1984;5(2):150-7.

13. Wilson BW, Arrieta DE, Henderson JD. Monitoring cholinesterases to detect pesticide exposure. Chemico-Biological Interactions. 2005;(15-158): 253-6. Doi: 10.1016/j.cbi.2005.10.043.

14. Furman J. Cholinesterase monitoring for agricultural pesticide handlers. Guidelines for health care providers in Washington state. Department of Labor \& Industries. WISHA Policy \& Technical Services. January 2006.

15. Richter ED, Rosenvald Z, Kaspi L, Levy S, Gruener N. Sequential cholinesterase tests and symptoms for monitoring organophosphate absorption in field workers and in persons exposed to pesticide spray drift. Toxicol Lett. 1986;33(1-3):25-35. Doi: 10.1016/0378-4274(86)90068-8.

16. Costa LG. Biomarker research in neurotoxicology: the role of mechanistic studies to bridge the gap between the laboratory and epidemiological investigations. Environmental Health Perspectives. 1996, Vol 104. Doi: 10.2307/3432696.

17. Quandt SA, Hernández-Valero MA, Grzywacz JG, Hovey JD, Gonzales M, Arcury TA. Workplace, household, and personal predictors of pesticide exposure for farmworkers. Environ Health Perspect. 2006;114(6):943-52. Doi: 10.1289/ehp.8529.

18. Lamadrid Boada AI, Romero Aguilera I, González Mesa III JE, Mandina Cardoso T. Biomonitoreo de trabajadores expuestos a plaguicidas. Rev Cubana Invest Bioméd. 2011;30(2):235-44.

19. Piperakis SM, Kontogianni K, Piperakis MM, Marcos R, Tsilimigaki S. effects of pesticides on occupationally exposed humans. Scientific World J. 2006;(6):1211-20. Doi: 10.1100/tsw.2006.207.

20. Pastor S, Lucero L, Gutiérrez S, Durbán R, Gómez C, Parrón T, Creus A, Marcos R. A follow-up study on micronucleus frequency in Spanish agricultural workers exposed to pesticides. Mutagenesis. 2002;17(1):79-82. Doi: 10.1093/mutage/17.1.79.

21. Undeger $U$, Basaran N. Assessment of DNA damage in workers occupationally exposed to pesticide mixtures by the alkaline comet assay. Arch Toxicol. 2002;76:430-36. Doi: 10.1007/ s00204-002-0355-5.

22. Zeljezic D, Garaj-Vrhovac V. Chromosomal aberration and single cell gel electrophoresis (Comet) assay in the longitudinal risk assessment of occupational exposure to pesticides. Mu- tagenesis. 2001;16(4):359-63. Doi: 10.1093/ mutage/16.4.359.

23. Grover P, Danadevi K, Mahboob M, Rozati R, Saleha Banu B, Rahman MF. Evaluation of genetic damage in workers employed in pesticide production utilizing the Comet assay. Mutagenesis. 2003;18(2):201-5. Doi: 10.1093/mutage/18.2.201.

24. Pastor S, Gutiérrez S, Creus A, Xamena N, Piperakis S, Marcos R. Cytogenetic analysis of Greek farmers using the micronucleus assay in peripheral lymphocytes and buccal cells. Mutagenesis. 2001;16(6):539-45. Doi: 10.1093/ mutage/16.6.539.

25. Bull S, Fletcher K, Boobis AR, Battershill JM. Evidence for genotoxicity of pesticides in pesticide applicators: a review. Mutagenesis. 2006;21(2):93103. Doi: 10.1093/mutage/gel011.

26. Costa C, Teixeira JP, Silva S, Roma-Torres J, Coelho P, Gaspar J, Alves M, Laffón B, Rueff J, Mayan O. Cytogenetic and molecular biomonitoring of a Portuguese population exposed to pesticides. Mutagenesis. 2006;21(5):343-50.

27. Bolognesi C. Genotoxicity of pesticides: a review of human biomonitoring studies. Mutation Res. 2003(543):251-72. Doi: 10.1016/S1383-5742(03)00015-2.

28. Bolognesi C, Creus A, Ostrosky-Wegman P, Marcos R. Micronuclei and pesticide exposure. Mutagenesis. 2011;26(1):19-26. Doi: 10.1093/ mutage/geq070.

29. Floria Pancetti Vaccari. Noncholinesterase Effects Induced by Organophosphate Pesticides and their Relationship to Cognitive Processes: Implication for the Action of Acylpeptide Hydrolase. Journal of Toxicology and Environmental Health, Part B. 2007;(10):623-30. Citado en PubMed PMID: 18049927

30. Judit Marsillach, Lucio G. Costab, Clement E. Furlong. Protein adducts as biomarkers of exposure to organophosphorus compounds. Toxicology. 2013;(307):46-54. Doi: 10.1016/j.tox.2012.12.007

\section{Fuente de financiamiento:}

Esta investigación fue financiada por el Centro Nacional de Salud Ocupacional y Protección del Ambiente para la Salud del Instituto Nacional de Salud, como parte de la cartera de proyectos institucionales.

\section{Conflictos de interés:}

El autor declara no tener conflictos de interés en la elaboración ni publicación de este artículo.

\section{Correspondencia:}

Mg. T.M. Jaime Alonso Rosales Rimache jrosales@ins.gob.pe; jaimerosale@gmail.com Celular: (51 1) 944457898

Teléfono: (51 1) 7480000 anexo 7706 y 7725 